
\title{
Efeito de um protocolo baseado no
Método Pilates sobre mobilidade, equilíbrio e risco de quedas em idosas da comunidade: ensaio clínico
}

Effect of a protocol based on the Pilates

Method on mobility, balance and fall risk in

elderly community: clinical trial

FisiSenectus. Unochapecó Ano 7, n. 2 - Jul/Dez. 2019 p. $39-52$

\section{Laís Leite Ferreira. lais.l.ferreira@hotmail.com}

Mestre em Biociências Aplicadas à Saúde pela Universidade Federal de Alfenas (Unifal/MG). Especialista em Acupuntura pela Universidade Federal de Alfenas (Unifal/MG) e em Cinesiologia, Biomecânica e Treinamento Físico pela Universidade Estácio de Sá/MG. Fisioterapeuta pelo Centro Universitário do Sul de Minas (UNIS/MG).

Mônica Beatriz Ferreira. monica.ferreira@unis.edu.br

Mestre em Gerontologia pela Universidade Estadual de Campinas (Unicamp). Especialista em Gerontologia pela Universidade Federal de São Paulo/Escola Paulista de Medicina (Unifesp/EPM). Especialista em Gestão Pública pelo IF Muzambinho. Especialista em Piscina Terapêutica pela Universidade Cidade de São Paulo (Unicid). Fisioterapeuta pela Universidade de Alfenas (Unifenas).

\section{Resumo}

Introdução: entre os distúrbios ocorridos pelo processo de envelhecimento, o equilíbrio corporal pode ser afetado, o que gera grandes impactos na autonomia social, predispondo a queda e trazendo imobilidade corporal e medo de cair ao idoso. Entre as propostas de intervenção, surge o Método Pilates (MP) com o intuito de trabalhar e melhorar a força muscular, o equilíbrio e a postura. Objetivo: este estudo teve o propósito de analisar o efeito de um protocolo baseado no MP sobre a mobilidade, o equilíbrio e o medo de cair em idosas da comunidade. Materiais e Métodos: trata-se de um ensaio clínico controlado aleatorizado, do qual participaram 29 idosos do sexo feminino, sendo o grupo controle (GC) formado por 15 participantes e o grupo experimental (GE) por 14 participantes. Foram avaliados os dados sociodemográficos (idade, estado civil e escolaridade), os testes Timed up and Go (TUGT), Teste do Alcance Funcional (TAF) e a Escala de eficácia de quedas (FES-I-BRASIL). Após a intervenção com o protocolo baseado no MP, ambos os grupos foram reavaliados. Resultados: houve melhoras significativas no GE após a intervenção nos parâmetros do $\operatorname{TUGT}(p=0,003)$ e no FES-I-BRASIL $(p=0,027)$, mas o resultado não foi significativo no teste TAF $(p=0,237)$. Ao comparar o GC com o GE após a intervenção, verificaram-se diferenças significativas nos parâmetros $\operatorname{TUGT}(p=0,002)$ e TAF $(p=0,030)$. Conclusão: o protocolo utilizado baseado no MP evidenciou melhoras na mobilidade, no medo de cair e também no equilíbrio em idosas da comunidade, demonstrando ser um método efetivo para a diminuição dos riscos de queda.

\section{Palavras-chave}

Acidentes por quedas; Equilíbrio postural; Técnicas de exercício e de movimento.

\section{Fisißenectus}




\begin{abstract}
Introduction: among the disorders caused by the aging process, body balance can be affected, which provides great impacts on social autonomy, predisposing to fall, bringing body immobility and fear of falling to the elderly. Among the intervention proposals comes the Pilates Method (PM) in order to work and improve muscle strength, balance and posture. Objective: This study aimed to analyze the effect of a protocol based on PM on mobility, balance and fear of falling in elderly women in the community. Materials and methods: this was a randomized controlled clinical trial, 29 elderly women participated in the control group (CG) with 15 participants and the experimental group (EG) with 14 participants. Sociodemographic data (age, marital status and educational level), Timed up and Go (TUGT), Functional Reach Test (FRT) and Falls Efficacy Scale (FES-I-BRAZIL) were evaluated. After intervention with the PM based protocol, both groups were reevaluated. Results: there were significant improvements in SG after intervention in TUGT parameters $(p=0.003)$ and FES-I-BRAZIL $(p=0.027)$, but it was not significant in the FRT test $(p=0.237)$. And when comparing the CG with the SG after the intervention, we found significant differences in the TUGT ( $p=0.002)$ and FRT $(p=0.030)$ parameters. Conclusion: the protocol used in PM either shows better mobility, fear of falling and there is no balance in the elderly in the community, proving to be an effective method to reduce the risk of falls.
\end{abstract}

\title{
Keywords
}

Accidents by falls; Postural balance; Exercise movement techniques

\section{Introdução}

$\infty \times \infty \times \infty \times \infty \times \infty \times \infty \times \infty \times \infty \times \infty \times \infty \times \infty \times \infty \times \infty \times \infty$

Nas últimas décadas, diversas regiões do mundo passaram por um processo de transição demográfica no qual populações, tipicamente jovens e adultas, tornaram-se gradualmente envelhecidas $^{1}$. No Brasil ${ }^{2}$, o número de idosos com idade acima de 60 anos passou de 3 milhões em 1960 para 7 milhões em 1975, 14 milhões em 2002 e 20 milhões em 2010, um aumento de 600\% em 50 anos. Estima-se que, em 2020, tal número alcance 32 milhões.

0 envelhecimento pode ser entendido como um processo dinâmico e progressivo, caracterizado tanto por alterações morfológicas, funcionais e bioquímicas quanto por modificações psicológicas de modo progressivo, o que afeta a capacidade de funcionamento do corpo humano ${ }^{3}$.

0 processo de envelhecimento provoca alterações tanto no sistema sensorial quanto no sistema motor, o que influencia na diminuição da força muscular, da amplitude de movimento, da velocidade de contração muscular e da acuidade visual e auditiva e causa alterações posturais, que acarretam perda da mobilidade funcional e déficit de equilíbrio ${ }^{4,5}$.

0 déficit de equilíbrio em idosos ocorre provavelmente em função da perda de força dos membros inferiores e de uma redução do processo sensório-motor ${ }^{6}$. Isso porque o processo do equilíbrio depende da combinação da sensação vestibular e periférica da visão, dos comandos centrais das respostas neuromusculares, da força muscular e dos proprioceptores que mandam a informação ao encéfalo, localizando as diferentes partes do corpo em relação ao ambiente ${ }^{7}$.

Consequentemente, esses distúrbios do equilíbrio corporal proporcionam grandes impactos na autonomia social, reduzindo as atividades de vida diária, predispondo quedas e fraturas e trazendo imobilidade corporal e medo de cair ao idoso ${ }^{8}$.

Estima-se que as quedas no Brasil tenham uma incidência de $30 \%$ anualmente entre idosos. Essa taxa aumenta para $40 \%$ entre os idosos com mais de 80 anos e para $50 \%$ entre os que vivem em instituições de longa permanência ${ }^{9}$. Além disso, as quedas também estabelecem um problema de saúde pública, uma vez que representam a sexta causa de óbitos em idosos, uma alta morbidade, além de estarem entre as principais causas de hospitalização das pessoas com 60 anos de idade ou mais ${ }^{10}$.

Entre a população idosa, tão importante quanto saber o real motivo das quedas, é propor condições que possam mudar esse cenário ${ }^{11,12}$. Entre as propostas de intervenção, surge o Método Pilates (MP) com o intuito de melhorar a força muscular, 
flexibilidade, postura e habilidades motoras do sujeito ${ }^{13}$.

O MP é uma técnica idealizada pelo alemão Joseph Hubertus Pilates durante a Primeira Guerra Mundial, amparado por seis princípios: concentração, consciência, controle, centro de força, respiração e movimento harmônico. É uma técnica dinâmica com benefícios como: alongamento, flexibilidade, força, estímulo sobre a circulação sanguínea, amplitude muscular, consciência corporal, coordenação motora, postura e equilíbrio ${ }^{14}$.

A técnica visa ao reforço dos músculos localizados no centro do corpo (abdominais, paravertebrais, glúteos e músculos do assoalho pélvico), denominados pelo criador Joseph Pilates de centro de força (powerhouse). Contudo, ocorre a estabilização dos músculos posteriores, anteriores e pélvicos, mantendo, portanto, o alinhamento da coluna vertebral adequado contra a gravidade e fornecendo suporte para movimentos dos mem$\operatorname{bros}^{13,14}$.

Estudos vêm demostrando que, quando o MP é aplicado em idosos, favorece a função pulmonar ${ }^{15}$, 0 aumento da força muscular ${ }^{16}$, a mobilidade, a coordenação ${ }^{17}$ e o equilíbrio ${ }^{18}$. No entanto, constam na literatura científica resultados controversos quanto aos objetivos e aos resultados obtidos com o MP em idosos, devido à baixa qualidade metodológica dos estudos ${ }^{19}$. Portanto, este estudo teve o propósito de analisar o efeito de um protocolo baseado no MP sobre o equilíbrio e o risco de quedas em idosas da comunidade.

\section{Métodos}

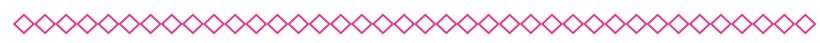

\section{Tipo de estudo e local}

Trata-se de um ensaio clínico controlado aleatorizado, composto por 30 mulheres idosas matriculadas no Projeto Unis Sênior do Centro Universitário do Sul de Minas (UNIS/MG). 0 estudo foi aprovado pelo Comitê de Ética em Pesquisa da Fundação de Ensino e Pesquisa do Sul de Minas Gerais (Fepesmig), com o número do parecer 1.870.542 e CAAE 62635716.2.0000.5111.
Os critérios de inclusão das amostras foram: possuir 60 anos ou mais; ter histórico de quedas ou risco para quedas, e/ou quase-quedas, como tropeços, tanto no domicílio quanto na rua; ter frequência mínima de $80 \%$ no treinamento; e ter assinado o Termo de Consentimento Livre e Esclarecido (TCLE). Já os critérios de exclusão foram apresentar sequelas neurológicas, motoras, déficit de compreensão e histórico de vertigem e ter a experiência prévia com exercícios baseados no MP.

As participantes foram aleatoriamente divididas em dois grupos, através de um sorteio on-line de numeração, sendo o número 1 para o grupo experimental (GE), totalizando 15 participantes que receberam a intervenção, e o número 2 para o grupo controle (GC), que também contou com 15 participantes, as quais não receberam a intervenção.

Os sujeitos do GC receberam as informações quanto à proposta da pesquisa e aguardaram o término do período da intervenção. Após o término do período deste estudo, a pesquisadora comprometeu-se a submeter o protocolo de exercícios a quem tivesse interesse.

A coleta de dados foi iniciada após a formação dos grupos, momento em que, primeiramente, ambos os grupos responderam ao questionário de caracterização dos sujeitos para obter os dados sociodemográficos (idade, sexo, estado civil e escolaridade). Posteriormente, foram submetidos a uma avaliação através dos testes Timed Up and Go Test (TUGT) ${ }^{20}$, Teste de Alcance Funcional (TAF) ${ }^{21}$ e da Escala de eficácia de quedas - Internacional - Brasil (FES-I-BRASIL) ${ }^{22}$, com pesquisadores cegos, sendo alunos de Fisioterapia treinados para a aplicação. Após a aplicação da intervenção, ambos os grupos foram reavaliados pelos mesmos testes.

\section{Timed Up and Go Test}

O Timed Up and Go Test (TUGT) é um instrumento desenvolvido em 1991 como uma versão modificada do Get Up and Go Test e traduzido pela língua portuguesa ${ }^{20}$. Tem como objetivo principal avaliar a mobilidade e o equilíbrio funcional, consequentemente é utilizado para avaliar o risco de quedas em pacientes internados ou em ambiente comunitário.

O TUGT quantifica, em segundos, o tempo gasto por uma participante para realizar a tarefa de levantar-se de uma cadeira (com apoio de, 
aproximadamente, 46 centímetros de altura e braços de 65 entímetros de altura), caminhar 3metros, virar, caminhar de volta rumo à cadeira e sentar novamente. Um menor tempo indica melhor desempenho funcional, enquanto um maior tempo indica maior risco de quedas.

A cronometragem do TUGT é iniciada a partir do deslocamento da posição inicial (costas apoiadas na cadeira) e é parada somente quando o idoso voltar à posição inicial novamente, sentado com as costas apoiadas na cadeira.

O tempo considerado normal para a realização do teste em adultos saudáveis é de até 10 segundos, independentes e sem risco de quedas; para idosos da comunidade, até 12 segundos seria considerado um valor de normalidade. Já para idosos com deficiência ou frágeis, são esperados valores entre 11 e 20 segundos, com independência parcial e com baixo risco de quedas. Um valor acima de 20 segundos sugere que o idoso possui importante déficit da mobilidade física e apresenta risco de quedas. Este escore foi adotado para esta pesquisa.

\section{Teste do Alcance Funcional}

0 Teste do Alcance Funcional (FRT) ${ }^{21}$ foi criado com o objetivo de identificar e avaliar as alterações dinâmicas do equilíbrio. Esse teste consiste em solicitar que as participantes fiquem em pé, com o ombro direito próximo a uma parede, onde foi colocada uma fita métrica, e que realizem uma flexão anterior do braço a $90^{\circ}$ com os dedos da mão estendidos. Nessa posição, o comprimento do membro superior direito do paciente é registrado na régua. Após esse procedimento, solicita-se ao paciente que tente alcançar algum objeto à frente, sem dar passos ou efetuar qualquer estratégia compensatória.

O resultado do teste é representado pela média, após três tentativas, da diferença entre a medida na posição inicial e na final registrada na régua. Deslocamentos menores que 15 centímetros indicam fragilidade do paciente e risco de quedas.

Escala de eficácia de quedas - Internacional - Brasil

A FES-I-BRASIL é uma adaptação da FES-I22, a qual foi possivel aplicar na população idosa da comunidade. Além disso, é o primeiro instrumento adaptado para a população brasileira no que diz respeito à avaliação do medo de cair em idosos. Por meio dessa escala, pôde-se avaliar o grupo com relação ao medo de cair durante a prática de 16 atividades diárias.

A escala apresenta questões sobre a preocupação com a possibilidade de cair ao realizar 16 atividades, com respectivos escores de 1 a 4 . 0 escore total pode variar de 16 (ausência de preocupação) a 64 (preocupação extrema), sendo que uma pontuação maior ou igual a 23 pontos ensejaria uma associação com queda esporádica, ao passo que uma classificação superior a 31 pontos ensejaria uma associação com queda recorrente.

\section{Método Pilates}

O Protocolo do MP empregado no GE foi baseado na série de exercícios seguindo Pata $^{23}$ e foi adaptado especificamente para a pessoa idosa de acordo com a Tabela 1 . 0 protocolo teve duração de 60 minutos, com frequência de duas vezes por semana, nas terças e quintas-feiras, durante oito semanas consecutivas, totalizando 16 sessões. Foram realizados os exercícios com as idosas sentadas e em pé, posicionadas na forma de círculo, as quais receberam o Theraband ${ }^{\circledR}$ para realizar os exercícios de resistência.

O controle respiratório foi orientado e incorporado durante todos os exercícios. As sessões iniciavam com um aquecimento de 10 minutos enfatizando a importância da respiração e da estabilidade do centro de força. Após o aquecimento, o protocolo de exercícios compreendia em torno de 25 minutos de exercícios ativos e de resistência dos segmentos de extremidade e melhora na flexibilidade da coluna vertebral na postura sentada.

Em seguida, os demais 15 minutos foram destinados à concentração de estabilidade e força muscular de membros inferiores. Para finalizar, os 10 minutos restantes foram empregados para desacelerar, concentrando na respiração e no equilíbrio.

\section{Análise dos Dados}

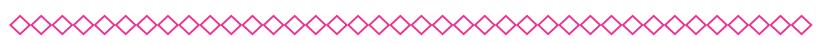

Os dados coletados foram submetidos, inicialmente, à análise estatística descritiva, com elaboração de tabelas de frequência para variáveis categóricas e medidas de posição e dispersão para variáveis contínuas. 
Em seguida, os dados foram analisados com o programa SigmaPlot, no qual, para a análise da normalidade da amostra, foi utilizado o teste de Shapiro-Wilk. Para a comparação intragrupos e intergrupos, foi utilizado o teste Student-T para as variáveis com distribuição normal, enquanto, para as variáveis que não apresentaram distribuição normal, utilizou-se o teste Mann-Whitney. 0 nível de significância de $5 \%(P \leq 0,05)$.

\section{Resultados}

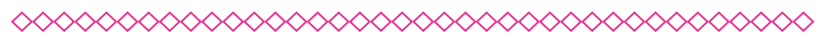

A amostra final da pesquisa contou com 29 idosas, pois uma participante foi excluída do GE por não participar assiduamente. Logo, o GC permaneceu com 15 idosas e o GE, com 14 idosas. A média de idade do GE foi de $69,6(7,4)$ anos e do GC, de 66,1 (5,7) anos, conforme a Tabela 2.

Os níveis de escolaridade tanto do GE quanto do GC apresentaram-se entre 9 e 12 anos de estudo, com $50 \%$ e $46,66 \%$, respectivamente. No estado civil, a maioria das participantes dos grupos caracterizou-se como viúva, sendo o GE com $42,85 \%$ e o GC com $53,33 \%$.

Após a intervenção, foi possível verificar diferenças estatisticamente significativas no GE em relação aos parâmetros avaliados, como na mobilidade com o teste TUGT $(P=0,003)$ e no medo de cair com a escala FES-I-BRASIL $(P=0,027)$. Porém, não houve diferença significativa no equilíbrio com o teste $\operatorname{TAF}(P=0,237)$, conforme apresentado na Tabela 3. No GC, cujos resultados constam na Tabela 4, não foi possível observar nenhuma diferença estatisticamente significativa.

Ao comparar a reavaliação do GE com o GC, verificou-se diferença significativa entre a mobilidade com o teste TUGT $(P=0,002)$ e o equilíbrio com o teste TAF $(P=0,030)$, no entanto, no aspecto medo de cair, não houve diferença significativa $(P=0,649)$, segundo consta na Tabela 5 .

\section{Discussão}

$\infty<\infty<\infty<\infty<\infty<\infty<\infty<\infty<\infty<\infty<\infty<\infty<\infty<\infty<\infty<\infty<\infty$

No presente estudo, evidenciou-se que, após a realização do protocolo do MP no GE, ocorreram melhoras significativas nos aspectos da mobilidade e do medo de cair, porém não houve alteração no equilíbrio. Ao comparar o GC com o GE após a intervenção, observou-se diferença significativa nos aspectos mobilidade e equilíbrio, mas não em relação ao medo de cair.

A queda é um problema crescente em idosos, que causa um alto grau de morbidade, mortalidade e procura pelos serviços de saúde; sendo assim, a identificação de fatores de risco, através de avaliações e intervenções apropriadas, é necessária para reduzir as taxas de quedas ${ }^{24}$. Evidenciou-se que, após a intervenção, todos os participantes melhoraram seus escores, o que pode excluir o risco para quedas das idosas.

A presente pesquisa baseou-se em um estudo que evidenciou significativas melhoras no equilibrio e na mobilidade, semelhantes aos resultados deste estudo, apontando que o protocolo é efetivo junto ao público idoso da comunidade ${ }^{23}$. Outro estudo analisou a eficácia do MP comparado aos exercícios tradicionais, envolvendo 31 participantes com risco de queda; em seus achados, demonstrou que o MP resultou em melhoras semelhantes às dos programas tradicionais ${ }^{25}$.

O MP é um recurso para melhorar o equilíbrio e a qualidade de vida dos idosos para o risco de queda na comunidade. O método é essencial para o fortalecimento dos membros inferiores, especialmente em idosos, o que pode contribuir para o equilíbrio postural, diminuindo a incidência de quedas devido à perda de massa associada ao processo de envelhecimento ${ }^{26,27}$.

Outro estudo que reforça a efetividade do MP analisou 63 mulheres idosas e realizou a comparação entre o MP, a facilitação neuromuscular proprioceptiva e o controle, observando-se melhoras no equilíbrio, tanto no MP quanto na facilitação neuromuscular, comparados ao grupo controle. No entanto, não houve diferenças entre MP e grupos de facilitação neuromuscular proprioceptiva ${ }^{28}$. Neste estudo, o grupo com intervenção também obteve melhoras se comparado com o grupo controle.

A exposição do MP em cinco semanas apresentou ganhos significativos em força muscular, e foi possível verificar a manutenção dos ganhos após 12 meses. Esses resultados permitem inferir que, embora realizado em um período curto de intervenção, o método contribuiu efetivamente para os ganhos necessários para prover um envelhecimento 
saudável e independente ${ }^{27}$, o que reforça o presente estudo, pois, após 16 sessões, foi possível observar melhoras nos aspectos da mobilidade e no medo de cair.

Idosos que praticam o MP podem aumentar a estabilidade do centro de força e melhorar a seguridade no desempenho da marcha, amenizando, assim, o risco de quedas e evitando o ciclo da que$\mathrm{da}^{28}$, o que explica os achados deste estudo quanto à redução do escore do medo de queda após o MP. Portanto, o MP pode ser sugerido como intervenção complementar ou mesmo alternativa de programas tradicionais para os idosos, o que pode influenciar positivamente nas medidas de força, equilíbrio, desempenho funcional e quedas ${ }^{29}$.

Este estudo se limitou à quantidade da amostra, o que pode ter influenciado nas comparações intergrupos.

\section{Conclusão}

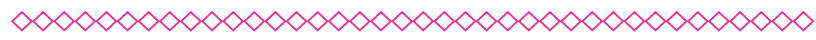

Neste estudo, o protocolo utilizado, baseado no MP, evidenciou uma melhora na mobilidade, no medo de cair e também no equilíbrio em idosas da comunidade, demonstrando ser um método efetivo para a diminuição dos riscos de quedas.

\section{Agradecimentos}

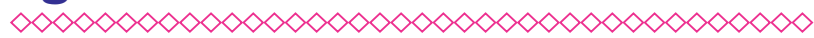

Os autores agradecem o Centro Universitário do Sul de Minas (UNIS/MG) e o apoio financeiro a bolsas de iniciação científica pela Fundação de Amparo à Pesquisa do Estado de Minas Gerais (Fapemig).

\section{Referências}

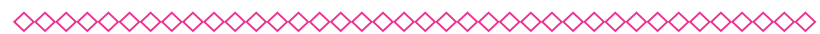

1. Miranda GMD, Mendes ACG, Silva ALA. 0 envelhecimento populacional brasileiro: desafios e consequências sociais atuais e futuras. Rev. Bras. Geriatr. Gerontol. 2016 mar;19(3):507-19.
2. Bezerra FC, Almeida MI, Nóbrega-Therrien SM. Estudos sobre Envelhecimento no Brasil: Revisão Bibliográfica. Rev. Bras. Geriatr. Gerontol. 2012 dez;15(1):155-67.

3. Ferreira OGL, Maciel SC, Costa SMG, Silva AO, Moreira MASP. Envelhecimento ativo e sua relação com a independência funcional. Texto Contexto Enferm. 2012 jul./set;21(3):513-8.

4. Navega MT, Furlanetto MG, Lorenzo DM, Morcelli MH, Tozim BM. Efeitos do método Pilates Solo no equilíbrio e na hipercifose torácica em idosas: ensaio clínico controlado randomizado. Rev. Bras. Geriatr. Gerontol. 2016 mar;19(3):465-72.

5. Oliveira AR, Gallagher JD, Oyen AS, Arruda GA, Greguol M. A influência da atenção seletiva no desenvolvimento do controle do equilíbrio: uma análise por acelerometria. Braz J Motor Behavior. 2010;5(Suppl):60.

6. Aveiro MC, Navega MT, Granito RN, Rennó ACM, Oishi J. Efeitos de um programa de atividade física no equilíbrio e na força muscular do quadríceps em mulheres osteoporóticas visando uma melhoria na qualidade de vida. Rev Bras de Ciência e Movimento. 2004;12(3):33-8.

7. Neto JP, Raso W, Brito CAF. Mobilidade funcional em função da força muscular em mulheres idosas fisicamente ativas. Rev Bras Med Esporte. 2015 set./out;21(5):369-71.

8. Sturnieks DL, St George R, Lord SR. Balance disorders in the elderly. Neurophysiol Clin. 2008;38:467-78.

9. Cunha AA, Lourenço RA. Quedas em idosos: prevalência e fatores associados. Rev. HUPE. 2014 abr./jun;13(2):21-9.

10. Antes DL, Schneider IJC, D'Orsi E. Mortalidade por queda em idosos: estudo de série temporal. Rev. Bras. Geriatr. Gerontol. 2015 Jul; 18(4):769-78.

11. Pestana MCS, Pestana VS, Pestana AMS, Schinoni MI. Comparison between the exercisesbased on pilates soil versus exercise resistance on the march and balance the elderl. Rev. Ciênc. Méd. Biol. 2013 Dez;12:441-8. 
12. Borges LL, Santos FPV, Pagotto V, Menezes RL. Functional disability in community-dwelling elderly: the role of cataracts and contextual factors. Fisioter. mov. 2014 Abr./Jun;27(2):189200.

13. Rodrigues BGS, Cader AS, Torre NVOB, Oliveira EM, Dantas EHM. Autonomia funcional de idosas praticantes de Pilates. Fisioterapia e Pesquisa. 2010 out./dez;17(4):300-5.

14. Di Lorenzo CE. Pilates: What Is It? Should It Be Used in Rehabilitation? Sports Health. 2011 Jul;3(4):352-61.

15. Roşu MO, Topa I, Chirieac R, Ancuta C. Effects of Pilates, McKenzie and Heckscher training on disease activity, spinal motility and pulmonary function in patients with ankylosing spondylitis: a randomized controlled trial. Rheumatol Int. 2014;34(3):367-72.

16. Fourie M, Gildenhuys GM, Shaw I, Shaw BS, Toriola AL, Goon DT. Effects of a mat Pilates programme on body composition in elderly women. West Indian Med J. 2013;62(6):524-8.

17. Lara S, Wendt P, Silva ML. Comparação da qualidade de vida em mulheres praticantes de Pilates e musculação. ConScientiae Saúde. 2014;13(1):134-40.

18. Guimarães ACA, Azevedo SF, Simas JPN, Machado Z, Jonck VTF. Efeito do método Pilates na flexibilidade de idosos. Fisioter Mov. 2014;27(2):181-8.

19. Engers PB, Rombaldi AJ, Portella EG, Silva MC. Efeitos da prática do método Pilates em idosos: uma revisão sistemática. Rev Bras Reumatol. 2016 mar;6(4):352-65.

20. Dutra MC, Cabral ALL, Carvalho GA. Tradução para o português e validação do teste timed up and go. Rev. Interfaces 2016 mar./maio; 3(9):81-8.

21. Duncan PW, Weiner DK, Chandler J, Studenski S. Functional reach: a new clinical measure of balance. J. Gerontol. 1990 Nov;45(6):192-7.

22. Camargos FFO, Dias RC, Dias JMD, Freire MTF. Adaptação transcultural e avaliação das propriedades psicométricas da Falls Efficacy
Scale - International em idosos brasileiros (FES-I-BRASIL). Rev Bras Fisioter. 2010 maio/ jun;14(3):237-43.

23. Pata RW, Lord K, Lamb J. The effect of Pilates based exercise on mobility, postural stability, and balance in order to decrease fall risk in older adults. Jour. of Bodywork \& Mov. Therap. 2013 Jul;18(3):361-7.

24. Cuevas-Trisan R. Balance Problems and Fall Risks in the Elderly. Phys Med Rehabil Clin N Am. 2017 Nov;28(4):727-37.

25. Josephs S, Pratt ML, Calk ME, Thurmond S, Wagner A. The effectiveness of Pilates on balance and falls in community dwelling older adults. J Bodyw Mov Ther. 2016 Jan;20(4):815-23.

\section{Souza ROB, Marcon LF, Arruda AAF, Pontes} Junior FL, Melo RC. Effects of Mat Pilates on Physical Functional Performance of Older Adults: a Meta-analysis of Randomized Controlled Trials. Sou J Phys Med Rehabil. 2017 Dez;97(6).

27. Regterschot GR, Folkersma M, Zhang W, Baldus H, Stevens M, Zijlstra W. Sensitivity of sensor-based sit-to-stand peak power to the effects of training leg strength, leg power and balance in older adults. Gait Posture. 2014 Ago;39(1):303-7.

28. Mesquita LSA, Carvalho FT, Freire LSA, Neto OP, Zângaro RA. Effects of two exercise protocols on postural balance of elderly women: a randomized controlled trial. BMC Geriatrics. 2015 Jun; 15(61):1-9.

29. Bird ML, Fell J. Positive long-term effects of pilates exercise on the age-related decline in balance and strength in older, communitydwelling men and women. Journal of Aging and Physical Activity. 2014 Jul;22(3):342-7.

30. Stivala A, Hartley G. The Effects of a PilatesBased Exercise Rehabilitation Program on Functional Outcome and Fall Risk Reduction in an Aging Adult Status-Post Traumatic Hip Fracture due to a Fall. Journal Of Geriatric Physical Therapy. 2014 Jul./Sept;37(3):136-45. 
31. Granacher U, Gollhofer A, Hortobágyi T, Kressig RW, Muehlbauer T. The importance of trunk muscle strength for balance, functional performance, and fall prevention in seniors: a systematic review. Sports Med. 2013 Jul;43(7):627-41.
32. Francisco CO, Fagundes AA, Gorges B. Effects of Pilates method in elderly people: Systematic review of randomized controlled trials. Journal of Bodywork \& Movement Therapies. 2015 Jul;19(3):500-8. 


\section{Anexos}

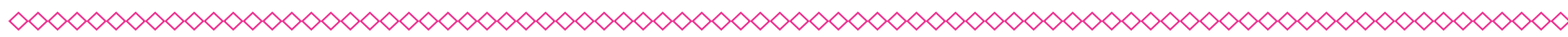

Tabela 1 - Protocolo de exercícios baseado no Método Pilates

\begin{tabular}{|c|c|c|}
\hline Exercício & Posição & Séries \\
\hline Respiração & $\begin{array}{l}\text { Inspirar pelo nariz, levando o ar para as partes } \\
\text { laterais e anteroposteriores da caixa torácica. Expirar } \\
\text { pela boca, contraindo os músculos abdominais, } \\
\text { levando o umbigo em direção às costas, para } \\
\text { estabilização da coluna lombar e pélvica. }\end{array}$ & $\begin{array}{l}\text { Contração mantida } \\
\text { durante todos os } \\
\text { exercícios. }\end{array}$ \\
\hline \multirow{21}{*}{$\begin{array}{l}\text { Sentado em } \\
\text { uma cadeira }\end{array}$} & 1- The Roll over* (Rolar) & $1 x$ de 10 repetições \\
\hline & 2- Respiração diafragmática & $\begin{array}{l}\text { 10x padrão } \\
\text { respiratório }\end{array}$ \\
\hline & $\begin{array}{l}\text { 3- Inspirar/expirar e acionar a musculatura do } \\
\text { assoalho pélvico e abdominal }\end{array}$ & $\begin{array}{l}\text { Condição em todas as } \\
\text { posturas de exercício. }\end{array}$ \\
\hline & 4- Shoulder rolls* (Rolar o ombro) & $1 x$ de 10 repetições \\
\hline & $\begin{array}{l}\text { 5- Estabilização das escápulas/realizar retração da } \\
\text { escápula com os ombros flexionados a } 90^{\circ}\end{array}$ & $1 x$ de 10 repetições \\
\hline & $\begin{array}{l}\text { 6- Exercícios de braços: inspire e direcione os braços } \\
\text { para cima em direção ao teto, expire e volte ao chão }\end{array}$ & $1 x$ de 15 repetições \\
\hline & $\begin{array}{l}\text { 7- Realizar flexão e extensão de pescoço: coloque o } \\
\text { queixo no peito e olhe para cima }\end{array}$ & $1 x$ de 10 repetições \\
\hline & 8- Exercícios de panturrilha apoiados no chão & $1 x$ de 10 repetições \\
\hline & 9- The hundred* (Cem) & $1 x$ de 10 repetições \\
\hline & 10- Abdução e adução das pernas com elas estendidas & $1 x$ de 10 repetições \\
\hline & 11- Roll up* (Enrolar) & $1 x$ de 10 repetições \\
\hline & $\begin{array}{l}\text { 12- One leg circles* (Movimento círculos de uma } \\
\text { perna) }\end{array}$ & $1 x$ de 10 repetições \\
\hline & $\begin{array}{l}\text { 13- Spine stretch forward* (Alongamento da coluna } \\
\text { para a frente) }\end{array}$ & $1 x$ de 10 repetições \\
\hline & 14- Bicycle* (Bicicleta) & $1 x$ de 10 repetições \\
\hline & 15- Rotação de tronco com extensão de perna junto & $1 x$ de 10 repetições \\
\hline & 16- Saw* (Serra) & $1 x$ de 10 repetições \\
\hline & 17- Swan* (Cisne) & $1 x$ de 10 repetições \\
\hline & 18- Neck pull* (Tração no pescoço) & $1 x$ de 10 repetições \\
\hline & 19- Swimming*: braços para cima e para baixo & $1 x$ de 10 repetições \\
\hline & $\begin{array}{l}\text { 20- Mermaid: estendendo-se o troco para um lado e } \\
\text { uma das mãos para o outro }\end{array}$ & $1 x$ de 10 repetições \\
\hline & 21- Sentar na beira da cadeira e estender uma perna & $1 x$ de 10 repetições \\
\hline \multirow[t]{4}{*}{ Em pé } & 1- Estender a perna lateralmente & $1 x$ de 10 repetições \\
\hline & $\begin{array}{l}\text { 2- Levantar a perna em todas as direções com a base } \\
\text { fechada, aberta ou em posição em "V" com os pés }\end{array}$ & $1 x$ de 10 repetições \\
\hline & 3- Alongamentos de panturrilhas & $1 x$ de 10 repetições \\
\hline & 4- Roll forward*: (alongamento para os isquiotibiais) & $1 x$ de 10 repetições \\
\hline
\end{tabular}




\section{Exercício}

\section{Posição}

Exercícios com 1- Rows* (Puxar o Theraband ${ }^{\circledR}$ em direção ao peito) Theraband $^{\circledR}$

2- Exercícios que trabalham bíceps

3- Exercícios que trabalham tríceps

4- Exercícios que trabalham Gastrocnêmios

5- Levantar os ombros e soltar

6- The hundred* (Cem)
$1 x$ de 5 repetições

\section{Séries}

$1 x$ de 5 repetições

$1 x$ de 5 repetições

$1 x$ de 5 repetições

$1 x$ de 5 repetições

$1 x$ de 5 repetições

*Nomenclatura dos exercícios do MP em inglês. Segue o protocolo de série de Pata, Lord e Lamb (2013) ${ }^{23}$.

(clique para voltar ao texto) 
Tabela 2 - Características sociodemográficas de anos de estudo e estado civil de ambos os grupos

\begin{tabular}{ccccc} 
Variável & & Grupo Experimental & Grupo Controle & P \\
& 1 a 4 & N $(\%)$ & $2(13,3)$ & \\
Anos de estudo & 5 a 8 & $3(28,57)$ & $4(26,7)$ & 0,454 \\
& 9 a 12 & $7(21,43)$ & $7(46,7)$ & \\
& 13 a mais & $0(0,0)$ & $1(13,3)$ & \\
\hline \multirow{2}{*}{ Estado civil } & Casada & $5(35,71)$ & $5(33,3)$ & \\
& Solteira & $1(7,14)$ & $0(0,0)$ & \\
& Divorciada & $2(14,28)$ & $2(13,4)$ & \\
& Viúva & $6(42,83)$ & $8(53,3)$ & \\
\hline
\end{tabular}

Nota: $\mathrm{n}$ = frequência absoluta; \% = frequência relativa; $\mathrm{P}$ = significância na comparação entre grupos.

(clique para voltar ao texto) 
Tabela 3 - Resultados dos testes do Grupo Experimental

\begin{tabular}{ccccccc}
\multicolumn{7}{c}{ Grupo Experimental } \\
& \multicolumn{7}{c}{ Avaliação } & \multicolumn{2}{c}{$\begin{array}{c}\text { Reavaliação } \\
\text { M }\end{array}$} & DP & M & DP & Diferença M \\
TUGT & 10,80 & 1,09 & 9,25 & 2,01 & 1,55 & $0,003^{*}$ \\
TAF & 22,86 & 6,51 & 25,93 & 6,90 & 3,07 & 0,237 \\
FES-I-BRASIL & 30,71 & 8,75 & 23,86 & 5,73 & 6,85 & $0,027^{*}$ \\
\hline
\end{tabular}

Nota: $\mathrm{n}$ = frequência absoluta; $\mathrm{M}=$ média; $\mathrm{DP}=$ desvio padrão; TUGT = Timed Up and Go Test; TAF = Teste de Alcance Funcional; FES-I-BRASIL: Escala de eficácia de quedas; ${ }^{*}=$ diferença estatisticamente significante intergrupos $(P<0,05)$. 
Tabela 4 - Resultados dos testes do Grupo Controle

\begin{tabular}{ccccccc}
\multicolumn{7}{c}{ Grupo Controle } \\
& \multicolumn{7}{c}{ Avaliação } & \multicolumn{1}{c}{$\begin{array}{c}\text { Reavaliação } \\
\text { M }\end{array}$} & DP & M & DP & Diferença M & P \\
TUGT & 12,50 & 4,90 & 12,04 & 3,71 & 0,46 & 0,786 \\
TAF & 20,90 & 4,40 & 20,04 & 5,20 & 0,86 & 0,983 \\
FES-I-BRASIL & 20,10 & 4,00 & 24,07 & 3,93 & 3,97 & 0,835 \\
\hline
\end{tabular}

Nota: $\mathrm{n}$ = frequência absoluta; $\mathrm{M}=$ média; $\mathrm{DP}=$ desvio padrão; TUGT = Timed Up and Go Test; TAF = Teste de Alcance Funcional; FES-I-BRASIL = Escala de eficácia de quedas; ${ }^{*}=$ diferença estatisticamente significante intergrupos $(\mathrm{P}<0,05)$. 
Tabela 5 - Comparação do Grupo Experimental com o Grupo Controle

\begin{tabular}{ccccccc} 
& $\begin{array}{c}\text { Grupo Experimental } \\
(n=14)\end{array}$ & \multicolumn{2}{c}{$\begin{array}{c}\text { Grupo Controle } \\
(\mathrm{n}=15)\end{array}$} \\
& \multicolumn{2}{c}{$\begin{array}{c}\text { Reavaliação } \\
\text { Reavaliação }\end{array}$} & $\begin{array}{c}\text { Diferença } \\
\text { M }\end{array}$ & p \\
TUGT & 9,25 & 2,01 & 11,30 & 3,50 & 2,05 & $0,002^{*}$ \\
TAF & 25,93 & 6,90 & 20,45 & 5,20 & 5,48 & $0,030^{*}$ \\
FES-I-BRASIL & 23,86 & 5,73 & 24,67 & 3,94 & 0,81 & 0,335 \\
\hline
\end{tabular}

Nota: $\mathrm{n}$ = frequência absoluta; $\mathrm{M}=$ média; $\mathrm{DP}=$ desvio padrão; TUGT = Timed Up and Go Test; TAF = Teste de Alcance Funcional; FES-I-BRASIL: Escala de eficácia de quedas; ${ }^{*}=$ diferença estatisticamente significante intergrupos $(P<0,05)$. 